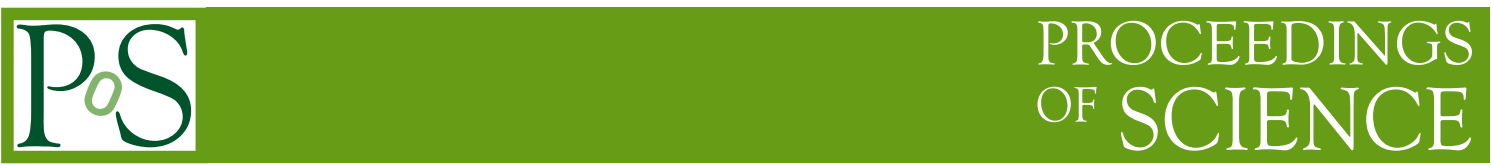

(Oscillating) non-exponential decays of unstable states

\title{
Francesco Giacosa*
}

Institut für Theoretische Physik, Goethe Universität, Max-von-Laue-Str. 1, 60438 Frankfurt am Main, Germany

E-mail: giacosa@th.physik.uni-frankfurt.de

\section{Giuseppe Pagliara}

Dipartimento di Fisica, Universitá di Ferrara and INFN Ferrara, via Saragat 1, 44100 Ferrara, Italy

E-mail: pagliara@fe.infn.it

\begin{abstract}
We discuss deviations from the exponential decay law which occur when going beyond the BreitWigner distribution for an unstable state. In particular, we concentrate on an oscillating behavior, remisiscent of the Rabi-oscillations, in the short-time region. We propose that these oscillations can explain the socalled GSI anomaly, which measured superimposed oscillations on top of the exponential law for hydrogen-like nuclides decaying via electron-capture. Moreover, we discuss the possibility that the deviations from the Breit-Wigner in the case of the GSI anomaly are (predominantely) caused by the interaction of the unstable state with the measurement apparatus. The consequences of this scenario, such as the non-observation of oscillations in an analogous experiment perfromed at Berkley, are investigated.
\end{abstract}

50th International Winter Meeting on Nuclear Physics

23-27 January 2012

Bormio, Italy

* Speaker. 


\section{Introduction}

The decay law of unstable systems plays a crucial role in Physics: the electromagnetic decays of atoms, the decays of radioactive nuclei, of hadronic resonances and of the Standard Model particles such as the weak interaction bosons and the Higgs boson, are all described by the well known exponential decay law. Once the decay rate $\Gamma$ is calculated from the microscopic interactions or measured in experiments, the decay law is simply given by $p(t)=e^{-\Gamma t}$, where $t$ is the time after the preparation of the unstable state and $p(t)$ represents the survival probability.

On the other hand, it is a fact that both in Quantum Mechanics [1] and in Quantum Field Theory $[2,3]$ a pure exponential decay law is not obtained: deviations from the exponential law are present at times very close to the initial preparation time $t=0$ and at very late times, while at "intermediate" times the exponential law represents a very good approximation. In particular, at late times the decay law follows a power-law, which is however very difficult to observe experimentally because it occurs at times for which the survival probability is already vanishingly small. On the other hand, the deviations at small times occur within a very short time scale, for instance $10^{-15}$ $\mathrm{s}$ for the electromagnetic decays of an excited hydrogen atom [4] and even shorter for hadronic decays [2]. It is thus experimentally very challenging to observe such deviations and to confirm the predictions of the theory. Only in 1997 cold atom experiments allowed to clearly observe for the first time deviations from the exponential decay law of unstable systems (via tunneling of atoms out of a trap) [5]. In particular, this experiment has shown that the survival probability at small times is not exponential, but it is rather a Gaussian, i.e. the derivative of $p(t)$ goes to zero at times close to the initial time, $p^{\prime}(0)=0$. In turn, this behavior allows for a quite peculiar modification of the decay law induced by measurements: when pulsed measurements on the system (inducing a collapse of the state into the original undecayed state) are performed during the non-exponential regime, one can obtain a slower or faster decay of the system depending on the frequency of the measurements. Those two effects, called Quantum Zeno and Anti-Zeno effects theoretically predicted in Refs. [6, 7, 8,9], have been then observed in the same experiment which has proven the existence of non-exponential decays [10]. This experimental success triggered a new interest of the physics community on the topic of deviations from the exponential decay law, not only because it represents a new and deep confirmation of the predictions of quantum theory, but also because it opens the possibility to engineer the decay of unstable quantum systems, see for instance Ref. [11]. Also, the general theory of measurement in quantum mechanics, which is still a quite active area of research, benefits from these experimental results [12].

In 2008, an experiment at the Storage Ring of the GSI facility of Darmstadt has reported the observation of non-exponential decays of hydrogen-like ions which decay via electron capture [13]. Quite remarkably, the survival probability shows an exponential decay with superimposed oscillations. These data stimulated many discussions and many different possible explanations have been proposed. Presently, there is no accepted theoretical explanation of this phenomenon and, more important, an experimental confirmation of the results is still lacking. By assuming that the phenomenon observed at GSI is real, we present a possible explanation in terms of "standard" quantum mechanical effects [14]. Moreover, we present further consequences of our explanation which can be proved or disproved in the near future. We also discuss the results obtained at the Berkeley Lab where no oscillations for the decays of the same nuclei have been observed [15]. 


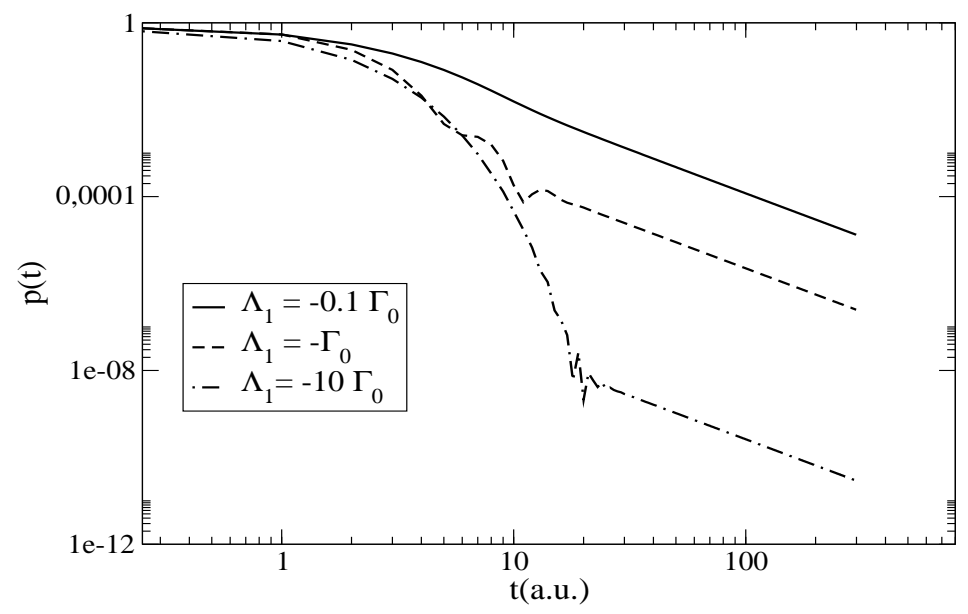

Figure 1: Survival probability for different values of the low energy cutoff $\Lambda_{1} . \Gamma=1, \Lambda_{2}=200 \Gamma$ (a.u.). The exponential decay law turns into a power law at large times.

\section{A phenomenological approach to unstable states}

The standard empirical approach to the decay of unstable states is to assume that the decay rate $\Gamma$, i.e. the number of decays per unit time, is constant and does therefore not depend from the "history" of the unstable system, in particular it does not depend on the time of "preparation" (in a quantum mechanical meaning) of the unstable state. This immediately leads to the exponential survival probability $p(t)=e^{-\Gamma t}$.

Denoting the energy distribution function (alias the spectral function) of the unstable state with $d(x)$ [1], the survival amplitude $a(t)$ is the Fourier transform of $d(x), a(t)=\int_{-\infty}^{\infty} \mathrm{dx} d(x) e^{-i x t}$, and the survival probability is just given by $p(t)=|a(t)|^{2}$. The "empirical" exponential decay law is theoretically justified, if we assume that $d(x)$ is a Breit-Wigner distribution $d(x) \rightarrow d_{B W}(x)=$ $\frac{1}{2 \pi} \frac{\Gamma}{(x-M)^{2}+\Gamma^{2} / 4}$, where $M$ is the mass of the unstable state (i.e. its energy in the rest frame) and $\Gamma$ its decay width. Note that $a(0)=1$ (the state is prepared at the instant $t=0$ with unit probability, $p(0)=1)$. When calculating the Fourier transform, the integral gets only the contribution of the simple pole located at $x_{\text {pole }}=M-i \Gamma / 2$ leading to $a_{B W}(t)=e^{-i M t} e^{-\Gamma t / 2}$ and thus $p_{B W}(t)=e^{-\Gamma t}$.

The exponential law works astonishingly well when compared to the experiments. However, there are two evident problems in assuming a Breit-Wigner spectrum: (i) It does not allow for the existence of a minimum of energy (threshold for the decay), i.e. it corresponds to an Hamiltonian unbound from below. (ii) The behavior of the Breit-Wigner at large energies is such that, while the normalization can be imposed (and thus unitarity), all the momenta of the distribution, including also the average energy of the unstable state, diverge. We need to cure these two problems in order to build a physically motivated distribution function. In Quantum Field Theory, once the interaction Hamiltonian between the unstable state/particle and the decay products is known, the spectral function is proportional to the imaginary part of the dressed propagator, i.e. the one obtained by the resumming all the loops, see Ref. [16]. This procedure allows to correctly describe the spectral function in the whole energy spectrum. In particular, there appears a threshold which then, in the survival probability, regulates the decay law of the system at large times, for which a power law is 
realized. The high energy behavior of the spectral function, which controls the survival probability at small times, represents unfortunately a much more complicated problem. All field theories are valid until a cutoff of energy where some new physical ingredients enter, for instance the Planck energy scale in particle physics. Following this reasoning, we construct here the simplest phenomenological model for the spectral function and, as a consequence, for the survival probability: we assume a Breit-Wigner spectrum corrected by two cuts in the energy, a low energy cut $\Lambda_{1}$ and a high energy cut $\Lambda_{2}$ :

$$
a(t)=N \int_{M-\Lambda_{1}}^{M+\Lambda_{2}} \mathrm{dx} \frac{\Gamma}{(x-M)^{2}+\Gamma^{2} / 4} e^{-i x t},
$$

where $N$ is such that $a(0)=1$. For such distribution, obviously all the momenta are finite (in particular the average energy of the unstable state). We show in Figs. 1 and 2 the effect of varying these two parameters on the survival probability $p(t)$ and on the decay rate as a function of time. The quantity $h(t)$ defined as $h(t)=-d p(t) / d t(h(t) d t$ represents the probability that the unstable state decays in the time interval between $t$ and $t+d t)$.

As expected, the exponential behavior dominates over a very long time scale but deviations are clearly present. The low-energy cutoff $\Lambda_{1}$ regulates the time after which the exponential law turns into a power law (in our approach the index of the power law cannot be adjusted, one should introduce another parameter which fixes how fast the decay rate falls to zero at threshold). When varying the high-energy cutoff $\Lambda_{2}$, the survival probability remains basically very similar to an exponential law (see Ref. [14] and figures therein), but very interesting features emerge in the behavior of $h(t)=-p^{\prime}(t)$. The high energy cutoff regulates the behavior of the decay probability at small times after the preparation of the system and manifests itself (in the Fourier transform) as an oscillation superimposed to the exponential decay law. The larger the cutoff, the larger is the frequency of the oscillation and the smaller is the amplitude of the oscillation as one can notice in Fig. 2 (where, for simplicity, the choice $\Lambda_{1}=\Lambda_{2}$ has been made). The physical interpretation of this phenomenon is quite natural: $\Lambda_{2}$ determines the bandwidth of the continuum of states into which the unstable state can decay. The plots shown in Fig. 2 interpolate between the pure exponential decay which occurs in presence of a large bandwidth continuum and a pure oscillating probability which occurs in a system of two discrete levels $\left(\Lambda_{2}\right.$ very close to the average energy of the unstable system) where Rabi oscillations are obtained.

The physical origin of the cutoff(s) can be twofold: there can be "natural" cutoffs determined by the microphysics of the interaction of the unstable state and its decay products (as the low-energy threshold and the high-energy cutoff mentioned above), but there could be an "experimental" cutoff, which is caused by the interaction of the unstable system with the experimental apparatus that measures the decay. As we will discuss in the following, in the case of the GSI anomaly the experimental cutoff must dominate (i.e. it is the smaller one).

\section{The peculiar case of the GSI anomaly}

The interesting properties of the decay law explained before, especially the oscillating behavior of $h(t)$ shown in Fig. 2 emerging for "small" values of the cutoff(s) (one or two orders of magnitude larger than $\Gamma$ ), might have been already observed in experiments. In particular, we want 


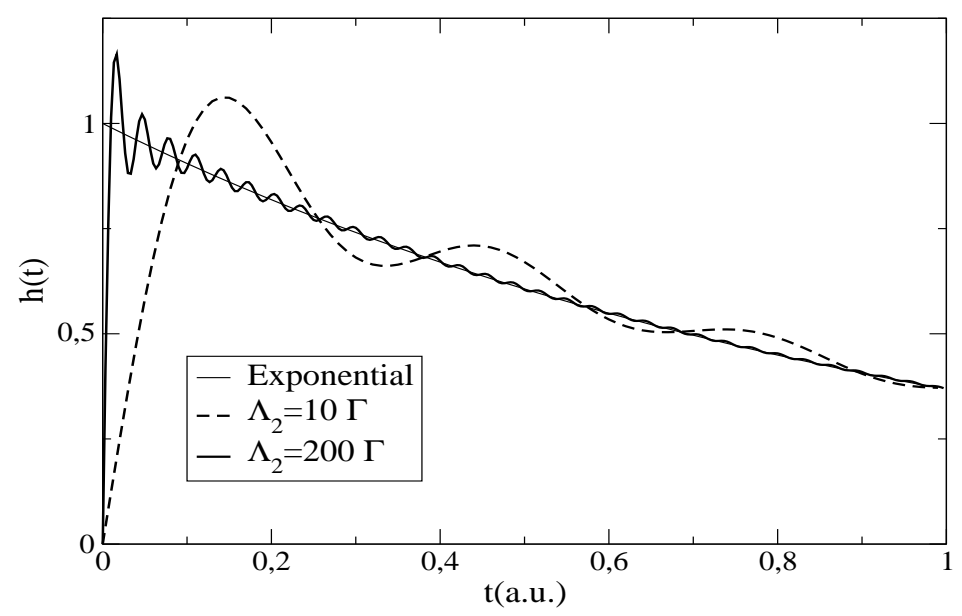

Figure 2: Decay rate as a function of time $h(t)$ for different choices of the high energy cutoff $\Lambda_{2} . \Gamma=1$, $\Lambda_{1}=\Lambda_{2}$ (a.u.). Also the exponential decay rate is shown for comparison.

to point to what has been named the "GSI anomaly" seen at the heavy ions storage ring at the GSI facility of Darmstadt. In 2008 Ref. [13] reported the observation of non-exponential decays of Hydrogen-like ions ${ }^{140} \mathrm{Pr}$ and ${ }^{142} \mathrm{Pm}$ in the electron-capture reactions of the form:

$$
M \rightarrow D+v_{e}
$$

where $M$ denotes the 'mother state' (i.e. the unstable $H$-like nuclide ${ }^{140} \mathrm{Pr}$ or ${ }^{142} \mathrm{Pm}$ ) and $D$ denotes the 'daughter state' (i.e. the nuclei ${ }_{58}^{140} \mathrm{Ce}$ and ${ }_{60}^{142} \mathrm{Nd}$, respectively).

Calling $N(t)$ the number of unstable particles at the instant $t$, it has been found that $d N / d t$ does not follow a simple exponential law. The experimental points were fitted with superimposed oscillations:

$$
\frac{d N_{d e c}}{d t}=-\frac{d N}{d t} \propto e^{-\lambda t}(1+a \cos (\omega t+\phi)),
$$

where $d N_{d e c} / d t$ represents the number of decay per time (see Fig. 3-5 of Ref. [13]). These results stimulated the theoretical modelling of this phenomenon, but the origin of these oscillations is not yet clear: explanations of the observed experimental data by invoking neutrino oscillations, neutrino spin precession and quantum beats seem indeed not to be satisfactory, see Refs. [17, 18, $19,20]$ and refs. therein.

In Ref. [14] we have put forward an interpretation of the GSI results based only on Quantum Mechanics: Following the discussions of Sec. II, we assumed that the mass distribution of the mother state is not a pure Breit-Wigner. In doing the calculations the survival probability amplitude of Eq. (2.1) with $\Lambda=\Lambda_{1}=\Lambda_{2}$ has been used. We have shown that for ${ }^{142} \mathrm{Pm}$ the cutoff $\Lambda \simeq 32 \Gamma \simeq$ $0.5 \cdot 10^{-15} \mathrm{eV}$, where $\Gamma=0.0224 \mathrm{~s}^{-1}$ is the decay width of the state, gives rise to oscillations which are qualitatively similar to those measured in Ref. [13].

The required value of the cutoff $\Lambda$ is very small. An intrinsic origin of this cutoff based on QED and QCD fundamental interactions, on the line of Ref. [4], seems very improbable in this case. A more promising direction consists in assuming that the cutoff originates from the interaction of the unstable system with the measuring apparatus. Indeed, the experiment performed at 
the GSI storage ring is unique. After their "creation", the unstable Hydrogen-like ${ }_{59}^{140} \mathrm{Pr}$ and ${ }_{61}^{142} \mathrm{Pm}$ ions are stored in a ring equipped with a Shottky Mass spectrometer which measures the frequency of rotation of the ions inside the ring. This frequency depends on the charge to mass ratio of the ions. When the reaction (3.1) takes place, the charge to mass ratio, and thus the rotation frequency, change. In this way the experimentalists at GSI can monitor the decay of these unstable systems few seconds after their preparation and for a period of a couple of minutes. Some important features of this experiment are worth to be mentioned: While the ions need $\sim 0.5 \mu$ s to complete a turn in the storage ring, their frequency of rotation within the frequency spectrum is identified within averagely $\Delta t_{\text {resolution }} \simeq 200 \mathrm{~ms}$. This means that the 'measurement' of the state of the unstable ions does not occur at every turn ${ }^{1}$. This measurement is clearly not an 'ideal measurement' in the quantum mechanical sense, according to which the collapse of the wave function occurs instantaneously as soon as the wave function of the unstable system interacts/overlaps with the measurement apparatus (the projection postulate of Quantum Mechanics). We connect the cutoff $\Lambda$ entering into the expression (2.1) to the precision of the experiment through the time-energy uncertainty relation:

$$
\Lambda \sim \frac{1}{\Delta t_{\text {resolution }}} \sim 10^{-15} \mathrm{eV} .
$$

This number is remarkably close to the value (mentioned above) needed to describe the oscillation seen in the GSI experiment. It seems therefore that the possibility of an apparatus-induced cutoff is viable and deserves further discussion. Indeed, the fact that the measurement itself can modify the decay law of an unstable system has been already experimentally proven by the observation of the quantum Zeno and Anti-Zeno effects [10]. To our knowledge, the first theoretical work on this problem is Ref. [21] "Does the Lifetime of an Unstable System Depend on the Measuring Apparatus" and recently a new interest on this issue has grown, see Refs. [11,12] and refs. therein. In Ref. [21] it is analyzed how the decay of an unstable state into two particles is modified by the measuring apparatus such as a bubble chamber. A length scale $R$ is introduced and named "localization radius", which corresponds to the distance between the decay products beyond which the experimental apparatus can ascertain whether the system has decayed or not. In this scheme, the following formula for $p(t)$, formally identical to Eq. (2.1) for $\Lambda=\Lambda_{1}=\Lambda_{2}=w / 2$, has been obtained by studying the dependence of the lifetime of the unstable state from the experimental apparatus:

$$
p(t)=N\left|\int_{E_{R}-w / 2}^{E_{R}+w / 2} d E \frac{e^{-i E t}}{\left(E-E_{R}\right)^{2}+\gamma^{2} / 4}\right|^{2},
$$

where $N$ is a normalization constant, $E_{R}$ is the average energy (i.e. the mass $M$ ) of the unstable state, $\gamma$ the width of the state (i.e. $\Gamma$ ) if it is not disturbed by the measurement, and the cutoff $w$ is proportional to the ratio of relative velocity $v$ of the two decay products and the localization radius $R, w=v / R$. The interpretation of $w$ in the case of Ref. [21] is quite clear: it is related to the time needed by the measuring apparatus to destroy the correlation between the unstable state and its decay products and it is thus of the same type of Eq. (3.3). As discussed in that paper, for the

\footnotetext{
${ }^{1}$ Another important experimental limitation concerns the time interval which lasts between the disappearance of the frequency of the mother ion and the appearance of the frequency of the daughter ion in the frequency spectrum. This time interval of $900 \mathrm{~ms}$ and $1200 \mathrm{~ms}$ (for ${ }^{140} \mathrm{Pr}$ and ${ }^{142} \mathrm{Pm}$ respectively) is related to the cooling of the stream before it can be identified by the mass spectrometer.
} 
typical measuring apparatuses in particle physics (as for instance the bubble chamber) $w$ is very large: $10^{17}-10^{22} \mathrm{~s}^{-1}$ and therefore the exponential decay is obtained to a very good approximation in most of the cases. The arguments in Ref. [21] leading $w$ are analogous to the emergence of a cutoff $\Lambda=w / 2$ in our case.

Indeed, one should go beyond these qualitative considerations and build a detailed theoretical model for the interaction between the unstable ions and the measuring apparatus also in the case of the GSI experiment, but this represents a quite demanding effort which is left for future work. Moreover, it will be also important to study in detail the effect of the 'collapse' of the wave function in the case of the GSI experiment. As discussed in Ref. [22, 23], the non-occurrence of the Zeno effect (and therefore the clock is not reset at each measurement) would assure that the quantity measured in the experiment coincides (up to a normalization) with the function $h(t)=-p^{\prime}(t)$. These issues go at the very heart of Quantum Mechanics: in this sense, the GSI experiment could represent a wonderful way to directly investigate them.

As a next step we list the predictions and consequences which hold in the framework of our proposed interpretation.

(i) The curve $h(t)=-p^{\prime}(t)$ : Our theoretical function $h(t)$ (which represents the decay probability per unit time and unit ion and it is thus proportional to $\left.d N_{d e c} / d t\right)$ evaluated starting from Eq. (2.1) shows some peculiar differences w.r.t. the experimental fitting curve of Eq. (3.2). Our $h(t)$ vanishes for short times (a general feature due to the fact that $p^{\prime}(0)=0$ ), the first peak is more pronounced than the others and the oscillations are damped faster than the fitting curve in Eq. (3.2), see Fig. 2 and the detailed discussion and figures in Ref. [14].

(ii) $\beta^{+}$decay channel: The $H$-like ions under study at the GSI do not decay only via the electron-capture mechanism of Eq. (3.1), but decay (in both cases sizably) via a $\beta^{+}$decay: $M \rightarrow$ $D^{\prime}+e^{+}+v_{e}$, where $D^{\prime}$ refers to the $H$-like daughter state for this process. In the case of the $\beta^{+}$ decay a positron is emitted which is absorbed by the environment extremely fast. Thus, for the $\beta^{+}$-channel the corresponding cutoff turns out to be much larger than $10^{-15} \mathrm{eV}$ : the deviations from the exponential decay law are very small and thus unobservable in this channel (see Fig. 2 to 'see' the effect of an increased cutoff). This discussion is also useful to clarify the following point: at variance with the positron, in the electron-capture decay of Eq. (3.1) the emitted neutrino does not interact with matter and is therefore not responsible for the determination of a time scale. For a mathematical description of the two-channel case we refer to Ref. [24]. A detailed study of the two-channel problem using the formalism of Ref. [24] is also part of our outlook.

(iii) Berkeley-experiment: In the experiment performed at the Berkeley Lab [15] no oscillations in the decay law for ${ }^{142} \mathrm{Pm}$ in relation to the 'same' process of Eq. (3.1) have been observed. As already noticed in Ref. [25], there are peculiar differences from the GSI experiment w.r.t. the Berkeley one: in the latter, the atoms are not ionised and are inside a lattice, thus also phonons are emitted in the final state. However, in the framework of our interpretation, the crucial fact is that very soon after the electron capture of Eq. (3.1), a $K$-shell vacancy is formed and a photon is very soon emitted. Thus, just as in the previous case, $\Delta t$ is much shorter and, conversely, the cutoff is much larger in the Berkeley-experiment: $\Lambda_{\text {Berkeley }} \gg \Lambda$. The oscillations have a too small amplitude and period and cannot be observed. Moreover, the absence of oscillations at the Berkeley experiment is a further strong argument against an intrinsic cutoff emerging out of microscopic form factor. 
(iv) Independence of the period and amplitude on the employed $H$-like ion: In the framework of our interpretation, the cutoff $\Lambda$ is almost uniquely related to the measurement process and is therefore independent of the employed mother nuclide. Thus, the period and the amplitude of the superimposed oscillations, which are controlled by the cutoff only, are also expected to be comparable: this is indeed the case of the two ions studied in Ref. [13]. Notice that the same cutoff of Eq. (3.3) for both ions ${ }^{140} \mathrm{Pr}$ and ${ }^{142} \mathrm{Pm}$ corresponds to quite different ratios of $\Lambda / \Gamma$, which are $\sim 32$ and $\sim 470$ respectively. It is interesting that the measurement-induced cutoff can explain naturally these quite different ratios.

(v) Repetition of the experiment. If the GSI experiment is performed with an improved time resolution, we expect that the corresponding cutoff increases, see Eq. (3.3), and thus the period and the amplitude of the oscillations decrease, see Fig. 2 for a numerical example.

Finally, it should be stressed that, while the here described qualitative features are general, a quantitative analysis should go beyond the simple formula of Eq. (2.1). This will be possible once that, as mentioned above, a detailed study of the interaction of the system as a whole (unstable state plus measurement) will be undertaken.

\section{Conclusions}

In this work we have described deviations from the exponential decay law when the energy distribution is not a Breit-Wigner function. In particular, we have studied a modified energy distribution in which cutoffs on the left and on the right sides of the peak have been introduced. We have proposed that the oscillations seen in Ref. [13] in the electron-capture decay of $H$-like ions may originate from a similar modification of the energy distribution of the mother state [14]. Inspired by Ref. [21], we have linked through the time-energy uncertainty relation the physical origin of the cutoff $\Lambda$ to the time uncertainty of the measuring apparatus at GSI. It is quite remarkable that the cutoff obtained in this way, see Eq. (3.3), is of the same order needed to obtain the time modulation of $7 \mathrm{~s}$ measured in Ref. [13].

We have analyzed the consequences of our proposal: very much suppressed oscillations in the $\beta^{+}$decay-channel because of a much larger cutoff, which makes them unobservable: the standard decay law holds here; similarly, suppressed oscillations (and thus exponential decay law) in the electron-capture decay channel at the Berkeley experiment; a mild dependence of the period and amplitude on the unstable ion; more suppressed oscillations when the GSI experiment is repeated with an increased time resolution (period and amplitude decrease).

As an outlook for future works we mention the precise modelling of the measurement procedure and the detailed study of the two-channel problem.

\section{Acknowledgments}

G.P. acknowledges financial support from the Italian Ministry of Research through the program Rita Levi Montalcini. F. G. acknowledges useful discussions with F. Bosch, Y. Litvinov, N. Winckler, S. Bishop, and D. Shubina. 


\section{References}

[1] Fonda, L., Ghirardi, G. C., Rimini, A., Decay theory of unstable quantum systems, Reports on Progress in Physics, Volume 41, Issue 4, pp. 587-631 (1978).

[2] F. Giacosa and G. Pagliara, Deviation from the exponential decay law in relativistic quantum field theory: the example of strongly decaying particles, Mod. Phys. Lett. A 26 (2011) 2247 [arXiv:1005.4817 [hep-ph]].

[3] G. Pagliara and F. Giacosa, Non exponential decays of hadrons, Acta Phys. Polon. Supp. 4 (2011) 753 [arXiv:1108.2782 [hep-ph]].

[4] Facchi, P., Pascazio, S., Temporal behavior and quantum Zeno time of an excited state of the hydrogen atom Phys. Lett. A, 241, (1998) 139.

[5] S. R.Wilkinson, C. F.Bharucha, M. C.Fischer, K. W.Madison, P. R.Morrow, Q. Niu, B. Sundaram, and M. G. Raizen, Experimental evidence for non-exponential decay in quantum tunnelling Nature 387, 575 (1997).

[6] B. Misra and E. C. G. Sudarshan, The Zeno's Paradox in Quantum Theory Journal of Mathematical Physics 18, 756 (1977).

[7] P. Facchi and S. Pascazio, Unstable Systems and Quantum Zeno Phenomena in Quantum Field Theory Quantum Probability and White Noise Analysis 17, 222 (2002).

[8] H. Nakazato, M. Namiki and S. Pascazio, Temporal Behavior of Quantum Mechanical Systems, Int. J. Mod. Phys. B 10, (1996) 247.

[9] P. Facchi and S. Pascazio, Quantum Zeno dynamics: mathematical and physical aspects Journal of Physics A 41, 3001 (2008).

[10] M. C. Fischer, B. Gutiérrez-Medina and M. G. Raizen, Observation of the Quantum Zeno and Anti-Zeno Effects in an Unstable System Phys. Rev. Lett. 87, 040402 (2001).

[11] A.G. Kofman and G. Kurizki, Universal Dynamical Control of Quantum Mechanical Decay: Modulation of the Coupling to the Continuum, Phy.Rev.Lett 87, 270405 (2001).

[12] K. Koshino, A. Shimizu, Quantum Zeno effect by general measurements Phys. Rep. 412, 191 (2005).

[13] Yu. A. Litvinov et al., Observation of Non-Exponential Orbital Electron Capture Decays of Hydrogen-Like ${ }^{140}$ Pr and ${ }^{142}$ Pm Ions, Phys. Lett. B 664 (2008) 162 [arXiv:0801.2079 [nucl-ex]].

[14] F. Giacosa and G. Pagliara, Oscillations in the decay law: A possible quantum mechanical explanation of the anomaly in the experiment at the GSI facility, arXiv:1110.1669 [nucl-th].

[15] P. A. Vetter, R. M. Clark, J. Dvorak, K. E. Gregorich, H. B. Jeppesen, S. J. Freedman, D. Mittelberger and M. Wiedeking, Search for Oscillation of the Electron-Capture Decay Probability of Pm-142, Phys. Lett. B 670 (2008) 196 [arXiv:0807.0649 [nucl-ex]].

[16] F. Giacosa and G. Pagliara, On the spectral functions of scalar mesons, Phys. Rev. C 76 (2007) 065204 [arXiv:0707.3594 [hep-ph]].

[17] A. G. Cohen, S. L. Glashow and Z. Ligeti, Disentangling Neutrino Oscillations, Phys. Lett. B 678 (2009) 191 [arXiv:0810.4602 [hep-ph]].

[18] A. Gal, Neutrino magnetic moment effects in electron-capture measurements at GSI, Nucl. Phys. A 842102 (2010). 
[19] A. Merle, The GSI oscillation mystery, Prog. Part. Nucl. Phys. 64 (2010) 445 [arXiv:1004.2347 [hep-ph]].

[20] J. Wu, J. A. Hutasoit, D. Boyanovsky and R. Holman, Is the GSI anomaly due to neutrino oscillations? - A real time perspective, Phys. Rev. D 82, 045027 (2010) [arXiv:1006.5732 [hep-ph]].

[21] A. Degasperis, L. Fonda and G. C. Ghirardi, Does the Lifetime of an Unstable System Depend on the Measuring Apparatus Nuov. Cim. 21471 (1974).

[22] M. Hotta and M. Morikawa, Complete resolutions of the quantum Zeno paradox for outside observers, Phys. Lett. A, Volume 326, Issue 1-2, p. 32-41.

[23] F. Delgado, J. G. Muga, and G. Garcia-Calderon, Suppression of the Zeno effect for distant detectors, Phys. Rev. A 74, 062102 (2006).

[24] F. Giacosa, Non-exponential decay in quantum field theory and in quantum mechanics: the case of two (or more) decay channels, arXiv:1110.5923 [nucl-th].

[25] T. Faestermann, F. Bosch, R. Hertenberger, L. Maier, R. Kruecken and G. Rugel, Could the GSI Oscillations be Observed in a Standard Electron Capture Decay Experiment?, Phys. Lett. B 672 (2009) 227 [arXiv:0807.3297 [nucl-ex]]. 